\title{
Astrometric and timing effects of gravitational waves
}

\author{
Bernard F. Schutz ${ }^{1}$ \\ ${ }^{1}$ Albert Einstein Institute, D-14424 Potsdam, Germany
}

\begin{abstract}
Gravitational wave detection can be done by precision timing of millisecond pulsars, and (with less likelihood) by precision astrometry on distant objects whose light or radio waves pass through gravitational waves on their way to our observatories. Underlying both of these is the relatively simple theory of light propagation in spacetimes with gravitational waves, which is also the basis of interferometric gravitational wave detectors. I review this theory and apply it to the timing and astrometric methods of detection. While pulsar timing might even be the first way that we directly detect gravitational waves, light deflection by gravitational waves seems out of reach.
\end{abstract}

Keywords. gravitational waves,gravitational lensing, astrometry,pulsars: general

\section{Introduction}

Gravitational wave detection is a challenge that is being pursued using many different technologies suited to finding waves in many different wavebands, with frequencies ranging from tens of kilohertz down to the Hubble frequency $H_{0}$. Although all the periods of these waves are long compared to the time-resolution needed for conventional astrometry, there are at least two ways in which astrometric considerations are relevant to gravitational wave detection. The first way is the detection of gravitational waves through the precise timing of millisecond pulsars using timing arrays; the second is the possibility that gravitational waves crossing the path of light or radio waves from a distant object might influence the observed frequencies or angular positions strongly enough to be detected. In order to understand both possible effects I will review the principles of "beam detectors", gravitational wave detectors based on the propagation of electromagnetic radiation, and then apply what we learn to the two problems just mentioned.

Ground-based gravitational wave detection is a very active field now. The LIGO Scientific collaboration (LSC 2009) operates the two 4-km interferometers of the LIGO project (Abbott et al. 2009) and the smaller GEO600 detector, a combined development platform and high-frequency observatory (Grote and the LIGO Scientific Collaboration 2008). The 3-km VIRGO interferometer (Acernese et al. 2008) shares data with the LSC detectors and does joint analysis of that data. All three large detectors were operating at the long-sought goal of a broadband strain sensitivity of $10^{-21}$ in 2007 . The S6 observing run, due to start in the middle of 2009, has a non-negligible chance of making the first detection, if it can achieve a somewhat better sensitivity. Other detectors are in the planning stage, the most advanced of which is the 3-km LCGT cryogenically cooled detector in Japan (LCGT 2009). The existing long-baseline detectors will reach a sensitivity of around $10^{-22}$ when they upgrade to Advanced Detectors around 2016. In the medium future it is now possible to envision super-sensitive ground-based detectors, with ten times better sensitivity even than Advanced Detectors and with a range extending to high redshifts, as a result of the European Einstein Telescope (ET) design study (ETP 2009). 
With a network of at least three major detectors observing an event, it is possible to fully reconstruct the event's polarization, source location, and intrinsic amplitude. As broad-band detectors, the ground-based systems are going to be the workhorses of gravitational wave astronomy. They will be able to reconstruct waveforms, measure distances to merging binary systems of neutron stars and black holes, perform strong tests of general relativity, and explore the world of compact stellar objects out to high redshifts. But they will not be able to observe below a few $\mathrm{Hz}$, and this means that their sources will be stellar-mass objects, up to perhaps $10^{3} M_{\odot}$. More massive objects radiate only at lower frequencies.

For this reason the international community is also developing the LISA space-based gravitational wave detector (LISA 2009), which will have a sensitivity in the milliHertz range. With the geometry of an equilateral triangle, LISA will actually provide three interferometry signals, so it is in fact a complete network in space, able to reconstruct positions, polarizations, amplitudes, and distances. Currently scheduled for launch in 2010, LISA is a cooperation between NASA and ESA. The current decadal review of astronomy in the US may have a significant influence on LISA's launch date. Beyond LISA there is a strongly developing project in Japan called DECIGO (Kawamura et al. 2006), aimed at exploring the $0.1 \mathrm{~Hz}$ band between LISA and the ground-based projects. Beyond that, there is a NASA concept study of the Big Bang Observer (BBO 2009), which demonstrates that, with effort, it might be possible to detect directly the gravitational waves left over from the Big Bang.

LISA will have enormous sensitivity, being able to detect mergers of black holes with masses between $10^{3}$ and $10^{7} M_{\odot}$ out to very high redshifts. By making observations of small black holes falling into larger ones (so-called Extreme Mass-Ratio Inspirals, or EMRIs), LISA will provide detailed maps of the geometry of the central black holes of many galaxies, testing general relativity and proving (or disproving!) that the central objects really are black holes. Although LISA's launch is far away, the mere existence of the project has already stimulated an unprecedented amount of scientific research, particularly into stellar populations in galaxies and in clusters around central black holes, as well into the dynamics of galaxy mergers and their subsequent black-hole mergers.

Coming along strongly in recent years is the search for gravitational waves using pulsar timing, e.g. (Jenet et al. 2006). Since millisecond pulsars are such good clocks, their arrival times at Earth record the effects of gravitational waves. By cross-correlating a suite of stable pulsars it is possible to beat down their noise further and look for a systematic variation in arrival times, which - if it has a quadrupolar pattern on the sky - could be due to a strong gravitational wave. The method requires averaging over several years, so it is only suited to frequencies below about $10^{-8} \mathrm{~Hz}$. But in this range there are interesting sources, particularly binaries of supermassive black holes. When the SKA is constructed (Carilli and Rawlings 2004), the detection and study of gravitational waves in this frequency range will become routine. But even in the next five years it is possible that pulsar timing will be the first technique to directly detect gravitational waves.

Pulsar timing and interferometry on the ground and in space are all examples of beam detectors, where a beam of light or radio waves is used to measure the amplitudes of gravitational waves. They work on the basis of timing: even in interferometers, the primary effect of the waves is to change the round-trip light travel-time along each arm, so that when light returns to the interference point, the two beams have been shifted in time relative to one another. We will see how this works below. The remaining technique for detection is the search for random cosmological gravitational waves in the microwave background radiation. Here the effect being measured is in the polarization of the microwave radiation; small changes in polarization are imprinted on the waves 
as they decouple from the expanding but recombining hot plasma, so the effect measures the amplitude of the gravitational waves at the time of decoupling. The recently launched PLANCK spacecraft (PLANCK 2009) will measure polarization, but it is not clear whether it will be able to see the small effect.

\section{Beam Detectors}

All interferometers and timing experiments on gravitational waves can be understood by studying the effect of gravitational waves on a beam of light. For simplicity we start with a restricted geometry: the light beam is travelling along the $x$-axis and the gravitational wave along the $z$-axis. The wave is a plane wave moving perpendicular to the light-beam. The light is moving between two mirrors or detectors that are themselves freely-falling in the gravitational field.

The wave is described by the following metric in linearized theory and the TT-gauge (Schutz 2009)

$$
\mathrm{d} s^{2}=-\mathrm{d} t^{2}+\left(1+h_{+}\right) \mathrm{d} x^{2}+\left(1-h_{+}\right) \mathrm{d} y^{2}+\mathrm{d} z^{2}, \quad h_{+}=h_{+}(t-z),
$$

where $h_{+}$is the amplitude of the "+" polarization component of the wave; the orthogonal " $\times$ " component does not affect the light-beam in this geometry.

The properties of this gauge, which is essentially a choice of coordinates, are that the spatial coordinates are comoving with free particles, so that the end-points of the beam remain at fixed coordinate locations even as the wave passes. The time-coordinate of this gauge is proper time on clocks at rest, which is, therefore, the time measured by clocks on the end-points of the light beam.

The one-way travel-time of light along the $x$-direction is, to first order in $h_{+}$, just solved from the above equation by setting $\mathrm{d} s^{2}=0$ and $\mathrm{d} z^{2}=0$. This gives $\mathrm{d} t^{2}=\left(1+h_{+}\right) \mathrm{d} x^{2}$, or integrating:

$$
t_{\text {end }}=t_{\text {start }}+\int\left[1+\frac{1}{2} h_{+}(t(x))\right] \mathrm{d} x .
$$

The argument of $h_{+}$here is just $t$, since the integration takes place on $z=0$. But the relevant time $t$ is the time at which the light-beam is at the location $x$. So this is an implicit equation: we want to find $t$ but need it in the integrand.

The way to solve this implicit equation is to recall that $h_{+}$is small, so that we do not need corrections of order $h$ inside the argument of $h$. We can set $t(x)=x$ in this argument, which is the relation between $t$ and $x$ when the wave is not present. This allows the integral to be done. We can see the result most easily if we differentiate the arrival time with respect to the initial time:

$$
\frac{\mathrm{d} t_{\text {end }}}{\mathrm{d} t_{\text {start }}}=1+\frac{1}{2} h_{+}\left(t_{\text {end }}\right)-\frac{1}{2} h_{+}\left(t_{\text {start }}\right) .
$$

The travel time varies only because of the wave amplitude when the beam starts out and when it arrives. The wave amplitude in the intervening time is not relevant. This is an important conclusion to which we will come back when we discuss gravitational wave astrometry below. To measure gravitational waves with this one needs stable clocks at both ends of the light beam.

Of course, our geometry is restricted, but if the wave were travelling at an angle $\theta$ to the $z$-axis, the only thing that would change is that there would be a $\cos \theta$ factor in the wave amplitude: a wave travelling in the same direction as the beam has no effect on it.

This formula was used to look for a possible supermassive binary black hole in 3C66b, using timing effects on the pulsar B1855+09 (Jenet et al. 2004). The pulsar is the stable 
clock at the emitting location, and the observatory clock is the stable reference at the receiving location.

From this it is easy to see how pulsar timing works. The beam starts at the pulsar and arrives at the Earth. When the variations in arrival times of pulses are correlated between different pulsars, the amplitudes of the gravitational waves at the pulsars themselves will go away, because they are uncorrelated. But the amplitude at the Earth will correlate, and there will be the factor of $\cos \theta$ with respect to the direction of the wave at the Earth. This gives a characteristic sky pattern that can be used to dig further into the residual pulsar timing noise and clock noise at the detector.

In an interferometer the light is reflected back from the distant mirror, so that the relevant time is the two-way travel time along a path whose proper length is $L$ when there is no gravitational wave. In our geometry this is just, to first order,

$$
t_{\text {return }}=t_{\text {start }}+2 L+\frac{1}{2} \int\left[h_{+}\left(t_{\text {start }}+x\right)+h_{+}\left(t_{\text {start }}+L+x\right)\right] \mathrm{d} x .
$$

To use this one needs only a stable clock in one location, namely at the emitter. The observable is the variation with time in this travel time. Interferometers essentially provide their second arm as the stable clock: the effect one looks for is the difference in light-travel-time variations between the two arms.

If we adopt a general geometry, with the wave arriving at an angle $\theta$ to the $z$-axis, then we get the following three-term formula for the variation in the return time:

$$
\begin{aligned}
\frac{\mathrm{d} t_{\text {return }}}{\mathrm{d} t_{\text {start }}}=\frac{1}{2}\left\{(1-\sin \theta) h_{+}\left(t_{\text {start }}+2 L\right)-(1+\sin \theta) h_{+}\left(t_{\text {start }}\right)\right. \\
\left.+2 \sin \theta h_{+}\left[t_{\text {start }}+(1-\sin \theta) L\right]\right\}
\end{aligned}
$$

This formula was first derived by Estabrook and Wahlquist (Estabrook and Wahlquist 1975) to support their efforts to detect gravitational waves by timing the transponding time to solar-system spacecraft, which is another beam-detector method. The three-term signature helps to discriminate signals from noise.

By combining the three-term formula for the two arms of an interferometer one gets the general expression for the difference in the variation in the return times in the two arms, which translates into a phase shift in the light as it recombines. As soon as the second arm is introduced, one has to consider both polarizations, since the arm need not lie in the plane defined in our example by the beam and the wave directions. Ground-based detectors operate in the simple regime where the gravitational wavelength is much larger than the arm length $L$. This means that the time-arguments in Equation (2.5) can all be expanded around $t_{\text {start }}$. Timing accuracies must be very precise: Advanced Detectors will have to sense time-delays of only $10^{-27} \mathrm{~s}$ ! LISA, on the other hand, has arms that become comparable to a wavelength at the high-frequency end of its observing band, so that its response pattern to waves is more complex. This has to be taken into account in LISA data analysis. Its timing resolution is more relaxed: only $10^{-22} \mathrm{~s}$.

\section{Light Deflection by Gravitational Waves}

Gravitational waves produce more effects than just time-delays. They can also deflect the direction of travel of a light beam, which has led to suggestions that precision astrometry could perhaps detect waves, particularly if the waves pass through the light or radio beam when they are still strong, i.e. shortly after they have been emitted by a highly relativistic event like the merger of two black holes. Some initial studies of the effect, however, overestimated the size of the deflection. This was finally cleared up in a series of investigations by, among others, Damour, Schäfer, and Kopeikin (Damour and 
Esposito-Farèse 1998; Kopeikin et al. 1999; Kopeikin and Schäfer 1999; Blanchet et al. 2001; Kopeikin and Korobkov 2005). I shall here give a simplified treatment based on linearized light propagation.

We need more than time-delays for this kind of astrometry, which involves the deflection of light beams. So we need the propagation equation for null geodesics, which is basically the transport equation for the momentum 4 -vector $p^{\mu}$ of a photon moving through the wave. Consider the same geometry as before, where the photon moves along the $x$-axis and the wave along the $z$-direction. Then any change in the direction of the photon will be seen as a change in its momentum in the $z$-direction. The equation of propagation is

$$
\frac{\mathrm{d} p^{\mu}}{\mathrm{d} \lambda}=\Gamma_{\alpha \beta}^{\mu} p^{\alpha} p^{\beta},
$$

which leads at lowest order to

$$
\frac{\mathrm{d} p^{z}}{\mathrm{~d} t}=\frac{1}{2} p^{0} \frac{\mathrm{d} h_{+}}{\mathrm{d} t}
$$

This can be integrated from the start to the end, giving, again to lowest order, a deflection angle

$$
\Delta \Theta=\frac{\Delta p^{z}}{p^{z}}=\frac{1}{2}\left[h_{+}\left(t_{\text {end }}\right)-h_{+}\left(t_{\text {start }}\right)\right] .
$$

The important conclusion from this is that - just as in our earlier discussion of timedelays - the effect depends only on the amplitude of the wave at the end points, i.e. where the wave starts and where it is observed. It does not matter if the light beam travels through a strong-wave region in between. The effects of this region cancel out, and the residual depends only on the (presumed weak) wave field at the end-points of the beam.

This is, of course, just a discussion at the lowest order. We can expect that the amplitude of strong waves might come in at the next order. In particular, if the spacetime through which the light beam travels is curved, say by a Newtonian gravitational field, then there will not be complete cancellation. So we could try to estimate a reasonable size for effects of order $(G M / r) h_{+}$for a beam passing a distance $r$ from a radiating source of mass $M$ giving off (possibly strong) waves of amplitude $h_{+}$.

Now, for wave detection, the distance $r$ must exceed one wavelength $\lambda$ of the gravitational wave, for otherwise we are not in the wave zone. This can have a serious effect. The wavelength is determined from the natural frequency of the radiating mass, $f \sim$ $\left(G M / R^{3}\right)^{1 / 2} / 2 \pi \sim \lambda^{-1}$, where $R$ is the size of the source. Now, there is an upper bound on the amplitude, $h_{+}<(G M / r)(G M / R)$ (Schutz 2009). Combining these results gives

$$
h_{+}<\left(\frac{G M}{R}\right)^{5 / 2} \sim v^{5},
$$

where $v$ is a typical velocity inside the source. When multiplied by $G M / \lambda$ to get the size of the nonlinear effect, one gets in the end

$$
\Delta \Theta<h_{+} \frac{G M}{\lambda}<\left(\frac{G M}{R}\right)^{4} \sim v^{8} .
$$

Clearly these effects are smaller than the linear deflection in Equation (3.3). For a gravitational wave source like the Binary Pulsar, for example, this means that the maximum nonlinear effect that could be detected by this method is $10^{-24}$. There are of course intrinsically stronger sources, with shorter timescales and wavelengths, but they will be transient, so the chance of observing them are reduced.

If the waves that do the deflection are not from any localized source, but from a cosmological or astrophysical random background, the conclusion is not much different. 
The characteristic amplitude of a wave of frequency $f$ in a random background whose energy density is a fraction $\Omega_{\mathrm{gw}}$ of the cosmological closure density $\rho_{c}$ is

$$
h_{c}=\left(G \rho_{c}\right)^{1 / 2} f^{-1} \Omega_{\mathrm{gw}}^{1 / 2} / 2 \pi .
$$

If the background comes from inflation, and we normalize the observation to a period of 3 years or a frequency of $10^{-8} \mathrm{~Hz}$, then we get

$$
h_{c}=10^{-18}\left(\frac{\Omega_{\mathrm{gw}}}{10^{-14}}\right)^{1 / 2}\left(\frac{f}{10^{-8} \mathrm{~Hz}}\right)^{-1} .
$$

This still needs to be multiplied by $G M / R$ appropriate to some deflecting mass, so this will be small as well. Of course, it is likely at some frequencies that there are astrophysical backgrounds with higher energy densities, but even if we go up to $\Omega_{\mathrm{gw}}=10^{-6}$, the nucleosynthesis upper bound, we gain only four orders of magnitude in amplitude.

It follows that the prospects for astrometric observation of gravitational waves are very slim.

\section{References}

LSC (2009), Ligo scientific collaboration home page, URL http://ligo.org/.

B. P. Abbott, R. Abbott, R. Adhikari, et al., Reports on Progress in Physics 72, 076901 (2009).

H. Grote and the LIGO Scientific Collaboration, Classical and Quantum Gravity 25, 114043 (2008).

F. Acernese, M. Alshourbagy, P. Amico, F. Antonucci, S. Aoudia, P. Astone, S. Avino, L. Baggio, F. Barone, L. Barsotti, et al., Journal of Physics Conference Series 120, 032007 (2008).

LCGT (2009), Large-scale cryogenic gravitational-wave telescope project, URL http://www.icrr.u-tokyo.ac.jp/gr/LCGT.html.

ETP (2009), Einstein telescope, URL http://www.et-gw.eu/.

LISA (2009), Laser interferometer space antenna, URL http://www.esa.int/esaSC/120376_index_0_m.html.

S. Kawamura, T. Nakamura, M. Ando, N. Seto, K. Tsubono, et al., Class. Quantum Grav. 23, S125 (2006), URL http://stacks .iop.org/0264-9381/23/S125.

BBO (2009), Nasa vision missions,

URL http://universe.nasa.gov/program/vision.html\#big\%20bang\%20observer.

F. Jenet, G. Hobbs, W. van Straten, R. Manchester, M. Bailes, J. Verbiest, R. Edwards, A. Hotan, J. Sarkissian, and S. Ord, Astrophys. J. (2006), astro-ph/0609013.

C. Carilli and S. Rawlings, New Astronomy Reviews 48 (2004).

PLANCK (2009), Planck home page, URL http://www.rssd.esa.int/index.php?project=PLANCK.

B. F. Schutz, A First Course in General Relativity, 2nd edition (Cambridge University Press, 2009).

F. Jenet, A. Lommen, S. Larson, and L. Wen, Astrophys. J. 606, 799 (2004), arXiv:astro$\mathrm{ph} / 0310276$.

F. B. Estabrook and H. D. Wahlquist, Gen. Rel. \& Grav. 6 (1975).

T. Damour and G. Esposito-Farèse, Phys.Rev.D 58, 044003 (1998), arXiv:gr-qc/9802019.

S. M. Kopeikin, G. Schäfer, C. R. Gwinn, and T. M. Eubanks, Phys.Rev.D 59, 084023 (1999), arXiv:gr-qc/9811003.

S. M. Kopeikin and G. Schäfer, Phys.Rev.D 60, 124002 (1999), arXiv:gr-qc/9902030.

L. Blanchet, S. Kopeikin, and G. Schäfer, in Gyros, Clocks, Interferometers ...: Testing Relativistic Gravity in Space, edited by C. Lämmerzahl, C. W. F. Everitt, and F. W. Hehl (2001), vol. 562 of Lecture Notes in Physics, Berlin Springer Verlag, pp. 141-+.

S. Kopeikin and P. Korobkov, ArXiv General Relativity and Quantum Cosmology e-prints (2005), arXiv:gr-qc/0510084. 